\title{
Differential oxidation of phenolic acids by laccase in homogeneous and heterogeneous systems during humification reactions
}

Zavarzina A. ${ }^{1}$, Demin V. ${ }^{1}$, Belova O. ${ }^{2}$, Lisov A. ${ }^{1,2}$

${ }_{1}^{1}$ Faculty of Soil Science, Moscow State University, Moscow, Russia, zavarzina@mail.ru

${ }^{2}$ Institute of Biochemistry and Physiology of Microorganisms, Pushchino, Moscow Region, Russia

doi: 10.36291/HIT.2019.zavarzina.084

Oxidation and condensation of low molecular weight phenolic compounds (PC) in the presence of biocatalysts is considered an important pathway of humic substances (HS) formation and organic $C$ accumulation in soils [1]. Recently we have found occurrence of phenolic acids in lichens: p-hydroxybenzoic acid (HDB), syringic acid (SYR) and vanillic acid (VAN) were most abundant, while protocatechuic acid (PCAT) and caffeic acid (CAF) occur in trace amounts [2]. We hypothesized that phenolic acids detected in lichens may serve as precursors of HS at the pre-vascular stage, however their oxidative reactivities require investigation. In this work we studied oxidation of phenolic acids by laccase of lichen Solorina crocea both in homogeneous and heterogeneous systems. We have prepared solutions of individual phenolic acids as well as mixtures of SYR and VAN acids with $p$-HDB acid (mixture 1), PCAT acid (mixture 2), gallic (GAL) acid (mixture 3 ) and CAF acid (mixture 4). Concentration of each acid in the mixture was $2 \mathrm{mM}$. Reactions were carried out in $20 \mathrm{mM} \mathrm{Na}$-acetate buffer ( $\mathrm{pH} \mathrm{4.5)} \mathrm{within} 24$ hours with both free laccase and laccase immobilized on hydroxyaluminum-kaolinite. Phenolic acids were analyzed by RPHPLC as described in [2].
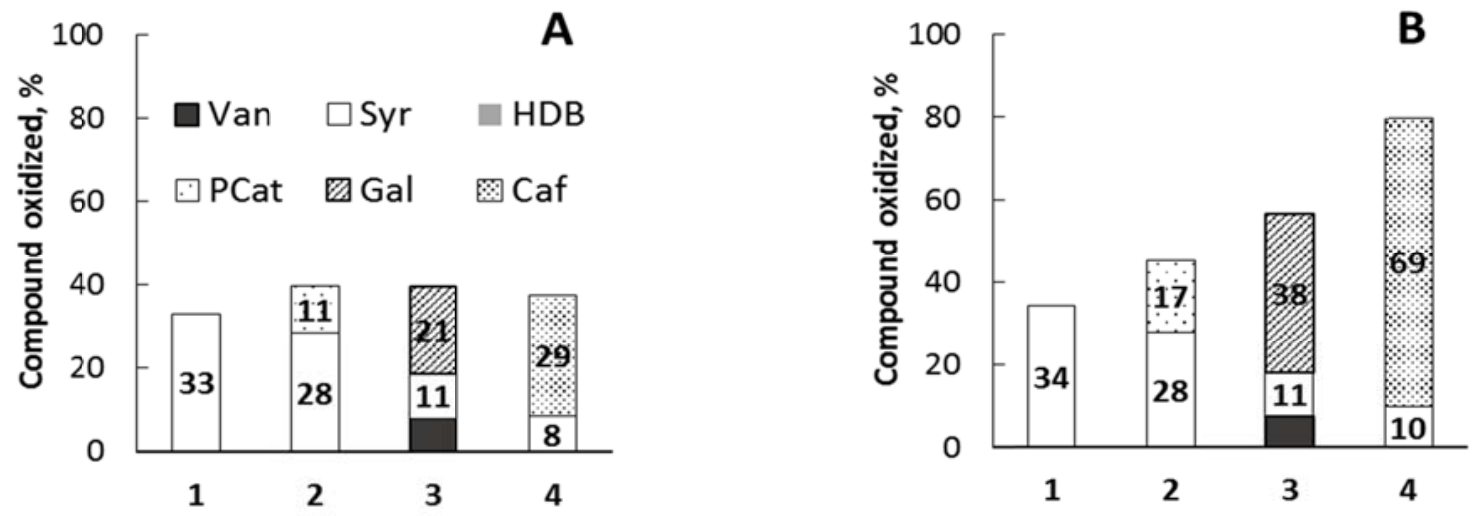

Fig. 1. Oxidation of phenolic acids mixtures by laccase in homogeneous $(A)$ and heterogeneous (B) systems.

We have found that oxidative reactivity of individual phenolic acids varied considerably and formed the order: GAL $\geq C A F>P C A T>S Y R>>V A N$. Hydroxybenzoic acid was not oxidized under the reaction conditions used. When present in mixtures, reactivity of $\mathrm{OH}$-substituted phenolic acids formed the order CAF>GAL>PCAT (Fig 1A). Methoxysubstituted VAN acid was oxidized only in the presence of GAL and SYR acids (mixture 3), while SYR acid was actively oxidized only in the absence of GAL and CAF acids (mixtures 1 and 2). Transformation of PCAT, GAL and CAF acids by laccase considerably increased in the presence of the mineral phase (Fig 1B) which is important finding in relation to aromatic $\mathrm{C}$ accumulation in soils.

Acknowledgements. The work was supported by RSF grant 17-14-01207.

References

1. Stevenson F.J. 1994. Humus chemistry: genesis, composition, reactions. J. Wiley and Sons, New York.

2. Zavarzina A.G. et al. // European J. Soil Sci. 2019. 1-15. doi: 10.1111/ejss.12822. 